\title{
Excessive Higgs pair production with little MET from squarks and gluinos in the NMSSM
}

\author{
Ulrich Ellwanger ${ }^{a, b}$ and Ana M. Teixeira ${ }^{c}$ \\ ${ }^{a}$ LPT, UMR 8627, CNRS, Université de Paris-Sud, \\ 91405 Orsay, France \\ ${ }^{b}$ School of Physics and Astronomy, University of Southampton, \\ Highfield, Southampton SO17 1BJ, U.K. \\ ${ }^{c}$ Laboratoire de Physique Corpusculaire, CNRS/IN2P3 - UMR 6533, \\ Campus des Cézeaux, 24 Av. des Landais, F-63171 Aubière, France \\ E-mail: Ulrich.Ellwanger@th.u-psud.fr, ana.teixeira@clermont.in2p3.fr
}

ABSTRACT: In the presence of a light singlino-like LSP in the NMSSM, the missing transverse energy - MET — signature of squark/gluino production can be considerably reduced. Instead, a pair of Higgs bosons is produced in each event. We propose benchmark points for such scenarios, which differ in the squark and gluino masses, and in their decay cascades. Events for these points are simulated for the run II of the LHC at $13 \mathrm{TeV}$ centre of mass energy. After cuts on the transverse momenta of at least four jets, and requiring two $\tau$-leptons from one Higgs decay, we find that the invariant mass of two $b$-jets from the other Higgs decay shows clear peaks above the background. Despite the reduced MET, this search strategy allows to see signals for sufficiently large integrated luminosities, depending on the squark/gluino masses.

Keywords: Supersymmetry Phenomenology

ARXIV EPRINT: 1412.6394 


\section{Contents}

1 Introduction 1

2 Scenarios with little MET in the NMSSM 3

3 Extraction of signals in $b \bar{b}+\tau^{+} \tau^{-}$final states $\quad 6$

4 Signals for benchmark points $\quad 8$

5 Summary and conclusions $\quad 11$

\section{Introduction}

After the first run of the LHC at a centre of mass (c.m.) energy of mostly $8 \mathrm{TeV}$, no significant excesses have been observed in searches for physics beyond the Standard Model (SM). Amongst others, this concerns searches for supersymmetric particles (sparticles) like squarks, gluinos, electroweak gauginos and higgsinos.

Lower bounds on the masses of sparticles have been obtained [1-3] which depend, however, on the sparticle decay cascades and hence on the complete sparticle spectrum. Recent summaries of bounds within various scenarios can be found in [4-7]. In particular, for similar squark ${ }^{1}$ masses $M_{\tilde{q}}$, gluino masses $M_{\tilde{g}}$ and decay cascades motivated by the Minimal Supersymmetric extension of the Standard Model (MSSM), ATLAS obtained $M_{\tilde{q}} \sim M_{\tilde{g}} \gtrsim 1.7 \mathrm{TeV}[8]$. Weaker limits are obtained within simplified models where, for instance, gluinos are assumed to be decoupled in the case of squark production. (Decoupled gluinos imply reduced squark production cross sections and, for similar squark and gluino masses, the largest production cross sections are the ones corresponding to one squark plus one gluino production.) In any case, these lower bounds have already put the MSSM under a certain stress.

Searches for squarks, gluinos and sparticles rely in general (assuming conserved Rparity) on events with large missing transverse energy $E_{T}^{\text {miss }}$ (MET) due to the escaping stable lightest supersymmetric particle (LSP), which is a good dark matter candidate if neutral. Since corresponding cuts on $E_{T}^{\text {miss }}$ are applied, these searches are less effective if, for kinematical reasons, the LSP produced in the last step NLSP $\rightarrow X+$ LSP of a sparticle decay chain is always soft and carries little energy. (NLSP denotes the Next-to-lightest supersymmetric particle.)

This is the case if the LSP is very light $\left(M_{\mathrm{LSP}} \sim\right.$ few GeV) and, simultaneously, $X$ corresponds to a Standard Model particle, with $M_{X}$ close to (just below) $M_{\mathrm{NLSP}}-M_{\mathrm{LSP}}$.

\footnotetext{
${ }^{1}$ Subsequently we use the notion "squark" for the scalar partners of the quarks of the first two generations; the scalar partners of top and bottom quarks will be denoted by stops and sbottoms, respectively.
} 
A possible Standard Model particle $X$ is the $125 \mathrm{GeV}$ Higgs boson, although additional non-SM-like Higgs bosons (or the $Z$ boson) could also play that role. In the general MSSM such a scenario cannot be realised in practise: whereas a bino-like LSP could be in principle very light and a wino- or higgsino-like NLSP have a mass close to $125 \mathrm{GeV}+M_{\mathrm{LSP}}$, most squarks (and sleptons) would then prefer to decay directly into the LSP, skipping the step NLSP $\rightarrow X+$ LSP. However, if this step is not present in nearly all sparticle decays, existing lower bounds on sparticle masses are hardly alleviated due to the other decay processes with an energetic LSP and large $E_{T}^{\text {miss }}$.

On the other hand, in extensions of the MSSM it is possible that the LSP has only weak couplings to all sparticles present in the MSSM. Then the MSSM-like sparticles (squarks, gluinos etc.) avoid direct decays into the LSP, but their decay cascades end up (provisionally) in the "MSSM-like LSP", typically the bino. Only subsequently does the "MSSM-like LSP" (now the NLSP) decay into the "true" LSP $+X$, always leading to a soft LSP for configurations of masses as stated above.

Scenarios of that kind have been discussed in [9-14]. The role of the "true" LSP can be played by a light gravitino (provided the decay of the MSSM-like LSP happens inside the detector - otherwise it behaves like the true LSP) [9, 11], so-called photini [12], or the singlino of the NMSSM [14].

The NMSSM denotes the Next-to-Minimal Supersymmetric extension of the Standard Model [15] where the coupling of the two Higgs doublets of the MSSM to an additional gauge singlet field $S$ solves the $\mu$-problem of the MSSM [16], and renders more natural a value of $\sim 125 \mathrm{GeV}$ of the SM-like Higgs boson [17-22], while preserving the attractive features of the MSSM. Besides the Higgs sector, the NMSSM differs from the MSSM through the presence of an additional neutralino (the singlino, the fermionic component of the singlet superfield). The singlino can be a light LSP, weakly coupled to the MSSM-like sparticles. A scenario with such a soft singlino-like LSP in the NMSSM was also briefly discussed in [10] and in a variant of the NMSSM including non-renormalisable terms in [9].

In [14] we studied in detail to which extent the reduction of $E_{T}^{\text {miss }}$ due to a light singlino in the NMSSM can alleviate the lower bounds on squark and gluino masses from the run I of the LHC. We presented a "worst case scenario" with all sparticle masses below $\sim 1 \mathrm{TeV}$, but consistent with constraints from the LHC. The dominant limits on such scenarios with little $E_{T}^{\text {miss }}$ actually originate from searches for many hard jets as in [23-26]. One particular feature of the scenario presented in [14] is that the role of $X$ is played by a NMSSM-specific Higgs boson lighter than $M_{Z}$ (but not ruled out by LEP). Such light Higgs bosons have very small branching ratios $(B R \mathrm{~s})$ into $W^{(*)} W^{(*)} / Z^{(*)} Z^{(*)}$ with subsequent leptonic decays of $W^{(*)}, Z^{(*)}$ leading to neutrinos. Neutrino decays generate $E_{T}^{\text {miss }}$, which makes corresponding scenarios somewhat more sensitive to standard supersymmetry search channels.

The production of Higgs bosons from neutralino cascades has been studied before in variants of the MSSM $[12,27-51]$ and the NMSSM [52-58]. The possible reduction of $E_{T}^{\text {miss }}$ due to a softer LSP in such decays was observed in [40, 41, 46, 57], but the emphasis was mainly on neutralino decays as additional sources of Higgs bosons. Since these can be considerably boosted, analyses of the substructure of the resulting jets have been proposed in $[45,59-61]$. 
In the present paper we concentrate, in contrast to [14], on the possible excessive production of pairs of Standard Model-like $125 \mathrm{GeV}$ Higgs bosons, $H_{S M}$. The "worst case scenarios" discussed in [14] relied, for reasons stated above, on the production of a lighter NMSSM-specific Higgs boson $H_{1}$ in the bino $\rightarrow X+$ singlino cascade and, moreover, it was assumed that squarks $\tilde{q}$ directly decay into quarks and the bino in order to alleviate as much as possible the constraints from searches based on $E_{T}^{\text {miss }}$ and jets. Here we study scenarios with longer squark decay cascades: squarks decaying via gluinos and/or gluinos decaying via stops/sbottoms. The final step in the decay cascades is always assumed to be bino $\rightarrow H_{S M}+$ singlino, hence $E_{T}^{\text {miss }}$ is still strongly reduced, making standard searches for supersymmetry less efficient. The aim is then to see whether signals of two Standard Modellike $125 \mathrm{GeV}$ Higgs bosons $H_{S M}$ can be extracted (above the Standard Model background) in order not to miss squark/gluino production at the $13 \mathrm{TeV}$ c.m. energy run II at the LHC.

To this end we present benchmark points with squark/gluino masses in the $1-1.5 \mathrm{TeV}$ range, which are not excluded by searches from run I. The benchmark points differ in the decay cascades; if stops/sbottoms appear therein, their masses are assumed to be $\sim 750 \mathrm{GeV}$ (the precise values of their masses have little impact on the final signatures). In each case we perform simulations and attempt to extract signals of two Standard Modellike $125 \mathrm{GeV}$ Higgs bosons $H_{S M}$ above the background, concentrating as in [14] on final states with $2 \tau^{\prime} s$ and a $b \bar{b}$ pair, the invariant masses of the latter near the Higgs mass.

In the next section we present the scenarios and the corresponding benchmark points in more detail. In section 3 we describe the simulations, the analysis and the dominant backgrounds. In section 4 we collect the results for the benchmark points and discuss which of their properties can help to distinguish the various scenarios. Section 5 is devoted to a summary and conclusions.

\section{Scenarios with little MET in the NMSSM}

As described in the Introduction and discussed in more detail in [14], a loss of $E_{T}^{\text {miss }}$ (associated to the LSP) in sparticle decay cascades can occur in the NMSSM if a dominantly singlino-like LSP is light, the mass of the typically mostly bino-like NLSP $M_{\mathrm{NLSP}}$ is somewhat above the sum of a Higgs and LSP masses $M_{\mathrm{NLSP}} \gtrsim M_{H}+M_{\mathrm{LSP}}$, and practically all sparticle decay cascades terminate by a last step NLSP $\rightarrow H+$ LSP. (Decays NLSP $\rightarrow Z+$ LSP occur only if the NLSP has a higgsino component, i.e. if the effective $\mu$ parameter $\mu_{\text {eff }}$ of the NMSSM [15] is relatively small, which we do not assume here for simplicity.)

The case where $H$ corresponds to a NMSSM-specific Higgs scalar with a mass below $M_{Z}$ was investigated in [14] (in order to reduce as much as possible $E_{T}^{\text {miss }}$ in all decay processes); here we consider the Standard Model-like $H_{125}$ and, accordingly, a bino-like NLSP mass somewhat above $125 \mathrm{GeV}$, as such scenarios are equally possible in the general NMSSM. The main purpose of the present paper is to propose and study benchmark points for squark/gluino production which, due to the reduction of $E_{T}^{\text {miss }}$, would be difficult to observe in standard supersymmetry search channels relying on large cuts on $E_{T}^{\text {miss }}$. Instead, 
we show that - for not too heavy squarks and large enough integrated luminosity — such scenarios are observable in searches for two Higgs bosons accompanied by hard jets.

The benchmark points considered here include scenarios where squarks decay via gluinos, leading to more jets in the final state but with reduced transverse momenta of the (s)particles involved in the last decay step. Gluinos lighter than squarks can undergo 3body decays into two quarks and a bino or, if kinematically allowed, 2-body decays into top-stop or bottom-sbottom pairs. Decay chains involving charginos or heavier neutralinos are left aside here as their decays via $W^{ \pm}$or $Z$ bosons can lead to neutrinos, and the $E_{T}^{\text {miss }}$ from the latter $\nu$ 's is often sufficient to make standard supersymmetry searches (possibly including isolated leptons) sensitive to these scenarios. Direct production of charginos or heavier neutralinos with subsequent Higgs pair production instead of $E_{T}^{\text {miss }}$ merits a separate analysis.

The subsequent benchmark points will not be defined in terms of parameters of the NMSSM but, for convenience, in terms of masses and branching fractions of the involved sparticles. However, these are chosen such that they can be reproduced at least approximatively by suitable parameters of the $\mathrm{NMSSM}^{2}$ as we have checked using NMSSMTools_4.4.0 [62, 63].

In [14] it was found that the loss of $E_{T}^{\text {miss }}$ is not very sensitive to the masses of the particles involved in the decay NLSP $\rightarrow H+$ LSP as long as $M_{\mathrm{NLSP}}-\left(M_{H}+M_{\mathrm{LSP}}\right) \ll$ $M_{\mathrm{NLSP}}$, and we verified that this also holds for the signals obtained below. Hence we do not vary these masses and choose for all benchmark points $M_{\mathrm{NLSP}}=130 \mathrm{GeV}, M_{H}=125 \mathrm{GeV}$, $M_{\mathrm{LSP}}=3 \mathrm{GeV}$ and $100 \% B R$ for the decay NLSP $\rightarrow H_{125}+\mathrm{LSP}$.

For the squark masses $M_{\tilde{q}}$ and gluino masses $M_{\tilde{g}}$ we choose values such that the benchmark points are not ruled out by searches at the run I at the LHC (using CheckMATE [64] for the standard supersymmetry search channels). All squarks of the first two generations are assumed degenerated. As stated above, the points differ by their squark $\rightarrow$ LSP decay chains, and for completeness we start with points for which this chain is as short as possible: decoupled stops and sbottoms, and gluinos only slightly heavier than - almost degenerate with - squarks. (Squarks much lighter than the gluino are unstable under radiative corrections and would imply an unnatural tuning of bare squark mass parameters.) The squark, gluino and stop/sbottom masses of the eight points P1 - P8 are shown in table 1, where we also include the sums of squark-squark, squark-antisquark, squark-gluino and gluino-gluino cross sections $\sigma_{\text {tot }}$ as obtained from Prospino at NLO [65, 66].

Points P1 and P2 are examples of short decay chains; for P1 the squark/gluino masses are slightly above the lower bounds from the LHC run I (i.e. $\sim 1 \mathrm{TeV}$, taken $\sim 150 \mathrm{GeV}$ heavier than in the "worst case scenario" studied in [14]) while the squark/gluino masses have somewhat more pessimistic values of $\sim 1.4 \mathrm{TeV}$ for P2. Both squark and gluino production contribute to the total production cross section. Gluinos heavier than squarks are assumed to decay democratically into all squark-quark pairs of the first two generations, and squarks lighter than gluinos with $100 \% B R$ into the bino-like NLSP and the

\footnotetext{
${ }^{2}$ This also ensures that all phenomenological constraints (except for the muon anomalous magnetic moment) tested in NMSSMTools are satisfied, in particular those from flavour physics (B meson decays).
} 


\begin{tabular}{|c|c|c|c|c|}
\hline Point & $M_{\tilde{q}}[\mathrm{GeV}]$ & $M_{\tilde{g}}[\mathrm{GeV}]$ & $M_{\tilde{t}}$ or $M_{\tilde{b}}[\mathrm{GeV}]$ & $\sigma_{\text {tot }}[\mathrm{fb}]$ \\
\hline P1 & 1000 & 1010 & decoupled & $\sim 1645$ \\
\hline P2 & 1400 & 1410 & decoupled & $\sim 168$ \\
\hline P3 & 1100 & 900 & decoupled & $\sim 1874$ \\
\hline P4 & 1500 & 1300 & decoupled & $\sim 169$ \\
\hline P5 & 1400 & 1410 & $M_{\tilde{t}}: 750$ & $\sim 168$ \\
\hline P6 & 1100 & 1110 & $M_{\tilde{b}}: 750$ & $\sim 920$ \\
\hline P7 & 1500 & 1300 & $M_{\tilde{t}}: 750$ & $\sim 169$ \\
\hline P8 & 1400 & 1200 & $M_{\tilde{b}}: 750$ & $\sim 321$ \\
\hline
\end{tabular}

Table 1. Squark, gluino, stop and sbottom masses (unless decoupled) and the sum of squark-gluino production cross sections at NLO for the benchmark points P1-P8.

corresponding quark: $\tilde{g} \rightarrow q \tilde{q} \rightarrow q \chi_{2}^{0}$.

Points P3 and P4 correspond to scenarios where gluinos are lighter than squarks. Now one can assume that the left-handed squarks fully decay into gluinos and the corresponding quarks $B R\left(\tilde{q}_{L} \rightarrow q \tilde{g}\right) \sim 100 \%$, while right-handed squarks, due to the larger hypercharge, have $B R$ of about $30 \%$ (assumed to be precise) into the bino-like NLSP and the corresponding quarks, leaving $70 \% B R$ into gluinos and the corresponding quarks, $B R\left(\tilde{q}_{R} \rightarrow q \chi_{2}^{0}\right) \sim$ $30 \%, B R\left(\tilde{q}_{R} \rightarrow q \tilde{g}\right) \sim 70 \%$.

For the remaining points $\mathrm{P} 5-\mathrm{P} 8$ we assume stops or sbottoms lighter than gluinos, in fact lighter than $M_{\tilde{g}}-m_{\text {top }}$ to allow for gluino 2-body decays. As before, gluinos can be slightly heavier or lighter than squarks, but squark and gluino masses have to be somewhat larger (depending on whether we have light stops or sbottoms) in order to be compatible with the limits from the run I of the LHC. For the same reason, stop or sbottom masses should be large enough. We observed that, as long as gluinos decay with $100 \% B R$ into top + stop or bottom + sbottom, the signals depend very little on the stop/sbottom masses, provided these are below $M_{\tilde{g}}-m_{\text {top }}$. Hence, instead of varying the stop/sbottom masses, we choose a sufficiently large value, $M_{\tilde{t}, \tilde{b}}=750 \mathrm{GeV}$ to comply with current LHC limits, but we neglect contributions from stop/sbottom pair production to the signals. These contributions are found to be very small (after the cuts discussed below) and would decrease even further for heavier stops/sbottoms; hence the signal rates also remain valid for heavier stops/sbottoms.

Points P5 and P6 correspond again to gluinos slightly heavier than squarks: for the gluino, $B R(\tilde{g} \rightarrow t \tilde{t}) \sim 100 \%(\mathrm{P} 5)$ or $B R(\tilde{g} \rightarrow b \tilde{b}) \sim 100 \%(\mathrm{P} 6)$, and squarks decay with $100 \% B R$ into the bino-like NLSP and the corresponding quark, $B R\left(\tilde{q} \rightarrow q \chi_{2}^{0}\right) \sim 100 \%$.

For the points $\mathrm{P} 7$ and P8, gluinos are lighter than squarks. Again, right-handed squarks are assumed to decay partially (with $70 \% B R$ ) into gluinos and the corresponding quark, with a $B R$ of $30 \%$ into the bino-like NLSP and the corresponding quark, but lefthanded squarks with $B R \sim 100 \%$ into gluinos. Herewith all relevant masses (summarised in table 1) and branching fractions of the benchmark points are defined. 

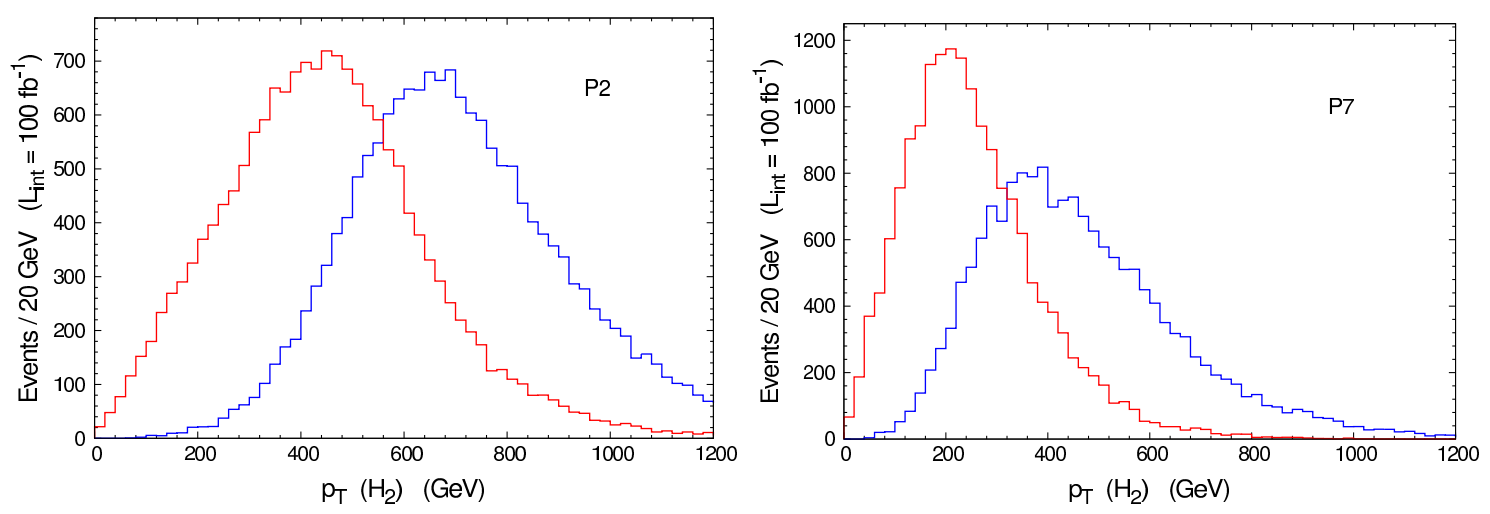

Figure 1. Spectra of the transverse momenta of the leading (blue) and next-to-leading (red) Higgs bosons for the benchmark points P2 (left panel) and P7 (right panel).

\section{Extraction of signals in $b \bar{b}+\tau^{+} \tau^{-}$final states}

Events due to squark/gluino production from $p p$ collisions at $13 \mathrm{TeV}$ are simulated using MadGraph/MadEvent [67] which includes Pythia 6.4 [68] for showering and hadronisation. The emission of one additional hard jet was allowed in the simulation; the production cross sections for the four distinct squark-squark, squark-gluino, squark-antisquark and gluinogluino production processes were obtained separately by Prospino at NLO [65, 66]. (The dominant contributions always come from squark-squark and squark-gluino production.) The output was given in StdHEP format to the detector simulation DELPHES [69]. Jets were constructed by Fastjet [70] (part of the Delphes package) using the anti- $k_{T}$ algorithm [71]. For $b$-tagged jets we require $p_{T}>40 \mathrm{GeV}$ and assume a $b$-tag efficiency of $70 \%$ (mistag efficiencies from $c$-jets of $10 \%$, and from light quark/gluon jets of $1 \%$ ).

For the analysis we try to profit from the fact that the events are rich in hard jets, and each event contains two Higgs bosons. A strong reduction of the Standard Model background - keeping the signal acceptance as large as possible — is achieved (see below) if we require that signal events contain at least two hadronically decaying $\tau$ leptons and at least two $b$-tagged jets.

The average transverse momentum of the Higgs bosons depends on the squark/gluino masses and, notably, on the length of the decay chains. This is clarified in comparing the spectra of the transverse momenta of the leading and subleading Higgs bosons of the points $\mathrm{P} 2$ and $\mathrm{P} 7$ in figure 1: the masses of the originally produced squarks and gluinos are similar $(\sim 1.4 \mathrm{TeV}$ for $\mathrm{P} 2,1.3 / 1.5 \mathrm{TeV}$ for $\mathrm{P} 7)$, but the transverse momenta of the leading Higgs boson peak near $700 \mathrm{GeV}$ for P2, where squarks decay directly into the NLSP, but somewhat below $400 \mathrm{GeV}$ for P7, in which one finds longer cascades, with the squarks decaying into gluinos and stops. (These analyses were performed by means of MadAnalysis 5 [72, 73].)

In principle, the decay products of strongly boosted Higgs bosons, as is the case of point P2, can be analysed using jet substructure methods [45, 59-61]. A similar approach, based on the construction of "slim" $R=0.15$ jets for each event, was employed in [14] where only scenarios with short squark decay cascades were considered. However, we found that 

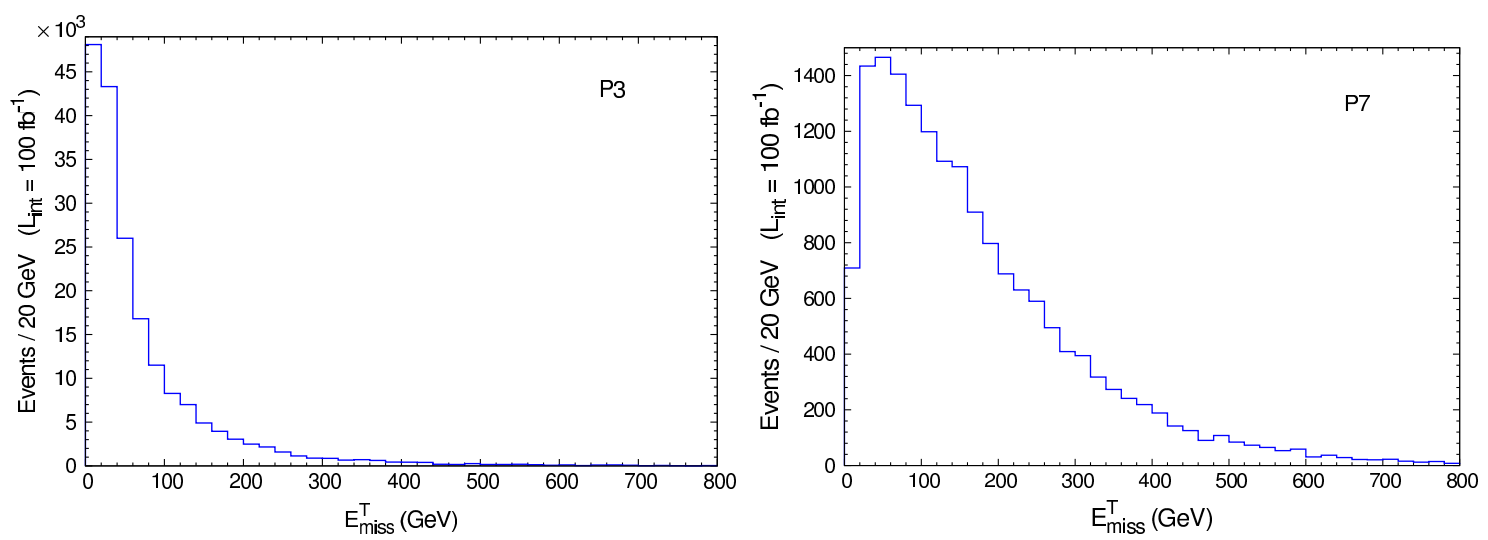

Figure 2. Spectra of $E_{T}^{\text {miss }}$ for the benchmark points P3 (left panel) and P7 (right panel).

the latter approach fails for scenarios with long squark decay cascades, which typically lead to less boosted Higgses. Instead, a more standard method leads to satisfactory results.

We construct jets with a jet cone radius $R=0.4$, and require at least two such jets to be $b$-tagged. Then we define the invariant mass $M_{b b}$ of the two $b$-tagged jets which are closest in $\Delta R$. (Muons inside such jets are added to the invariant mass of the system.) This simple approach works for all benchmark points; even the ones with large average transverse momenta of Higgs bosons lead to sufficiently many events with less boosted Higgses whose mass can be reconstructed this way.

Compared to analyses based on slim jets, the use of more standard $R=0.4$ jets has the additional advantage that the $\tau$ fake rate (and notably the unusually large 2- $\tau$ fake rate observed in [14]) is much smaller, which helps to suppress the background from QCD.

The spectra of $E_{T}^{\text {miss }}$ are quite soft for all the benchmark points due to the kinematical reasons discussed in the Introduction. Still, requiring two $\tau$ 's in the final state implies that some $E_{T}^{\text {miss }}$ will always be due to the escaping $\tau$ neutrinos. In addition, leptonic $b$-decays (from Higgs decays or cascades via stops/sbottoms) can generate some $E_{T}^{\text {miss }}$. Especially for cascades via top quarks, as in points $\mathrm{P} 5$ and $\mathrm{P} 7, E_{T}^{\text {miss }}$ from leptonic top decays can be relatively large. Finally, also for short decay cascades, the $E_{T}^{\text {miss }}$ spectrum becomes harder for heavier squarks and gluinos. Among the benchmark points, the softest $E_{T}^{\text {miss }}$ spectrum is observed for the point P3, the hardest for the point P7. Both are shown in figure 2. The point P7 could possibly also be discovered in standard search channels with sizeable cuts on $E_{T}^{\text {miss }}, E_{T}^{\text {miss }} \gtrsim 100 \mathrm{GeV}$, but if such cuts were imposed, then most of the events from points like P3 would be missed. Hence we use only a mild lower cut on $E_{T}^{\text {miss }}$ of $30 \mathrm{GeV}$.

In our analysis the following cuts were applied:

- At least 4 jets (b-tagged or not) with transverse momenta $P_{T}>400 \mathrm{GeV},>300 \mathrm{GeV}$, $>200 \mathrm{GeV}$ and $>100 \mathrm{GeV}$ for the 4 leading jets, respectively.

- At least two $b$-jets with $P_{T}>40 \mathrm{GeV}$ were required, and a small lower cut on $E_{T}^{\text {miss }}>$ $30 \mathrm{GeV}$ was applied. 
- At least two hadronically decaying $\tau$ 's are required, with invariant masses ranging from $20 \mathrm{GeV}$ to $160 \mathrm{GeV}$, the sum of their transverse momenta imposed to be above $100 \mathrm{GeV}$ (which further suppresses fake $\tau$ 's).

- Finally a (large) signal region $60 \mathrm{GeV}<M_{b b}<160 \mathrm{GeV}$ was defined; not only this allows to take into account uncertainties in the measurements of $M_{b b}$, but also to remain sensitive to possible additional Higgs bosons with masses below $125 \mathrm{GeV}$. (Additional Higgs bosons with masses below $60 \mathrm{GeV}$ must be practically pure singlets to avoid constraints from LEP, and to avoid decays of the $125 \mathrm{GeV}$ Higgs boson into such pairs which would reduce its observed signal rates. Then the couplings of such light additional Higgs bosons to a bino must be very small.)

Various SM backgrounds have been considered: top quark pair production, possibly together with 1-2 hard jets at the parton level; bottom quark pair production, also possibly together with 1-2 hard jets at the parton level; the production of four bottom quarks; direct production of $\tau$ 's from $Z$ bosons together with bottom or top quark pairs. All SM backgrounds get strongly reduced by the combined cuts on 4 hard jets (see above), two hadronically decaying $\tau$ 's, and $2 b$-quarks with an invariant mass in the signal region. The by far dominant contributions to the signal region were found to originate from top quark pair production together with 1 hard jet at the parton level (and possibly fake $\tau$ 's), and bottom quark pair production with 2 hard jets at the parton level (and two fake $\tau$ 's). We have simulated 300000 top pair production events and 500000 bottom pair production events using the same procedure as for the benchmark points. About $0.33 \%$ of the top pair events contained two $\tau$ 's satisfying the criteria of our cuts, while this only occurs for circa $0.03 \%$ of the bottom pair events. Finally we obtained contributions to the signal region of $\sim 0.029 \mathrm{fb}$ from top pair production and $\sim 0.031 \mathrm{fb}$ from bottom pair production, i.e. $\sim 0.06 \mathrm{fb}$ all together. The sum of all other background contributions to the signal region was found to be below $10^{-3} \mathrm{fb}$.

\section{Signals for benchmark points}

In this section we discuss the properties of the benchmark points, the signals of Higgs bosons as well as other observables which allow for hints on the underlying sparticle spectrum.

First, the spectra of $M_{b b}$ after the cuts described in section 3 are summarised in figure 3 for the points $\mathrm{P} 1-\mathrm{P} 4$. The cross sections in the signal region for these points are $\sim 3.1 \mathrm{fb}(\mathrm{P} 1), \sim 0.35 \mathrm{fb}(\mathrm{P} 2), \sim 1.3 \mathrm{fb}(\mathrm{P} 3)$ and $\sim 0.30 \mathrm{fb}(\mathrm{P} 4)$, i.e. all are well above the background cross section in the signal region of $\sim 0.06 \mathrm{fb}$. Comparing the signal cross sections with the production cross sections in table 1 , we see that the acceptances are about $1-2 \times 10^{-3}$, increasing with increasing squark/gluino masses. Note that, given the $B R\left(H_{125} \rightarrow 2 \tau\right) \sim 7 \%$ and $B R(\tau \rightarrow$ hadrons $) \sim 63 \%$, only $\sim 5.5 \%$ of all signal events contain 2 hadronic $\tau$ 's from Higgs decays. The efficiency for hadronic $\tau$ 's depends on their energy and their $P_{T}$ (improving with $P_{T}$ ), hence on the kinematics of their production. For the benchmark points we have an efficiency of $\sim 0.35 \ldots 0.65$. Hence the acceptances for the benchmark points of about $1-2 \times 10^{-3}$ are dominated by the requirement of 2 hadronic 

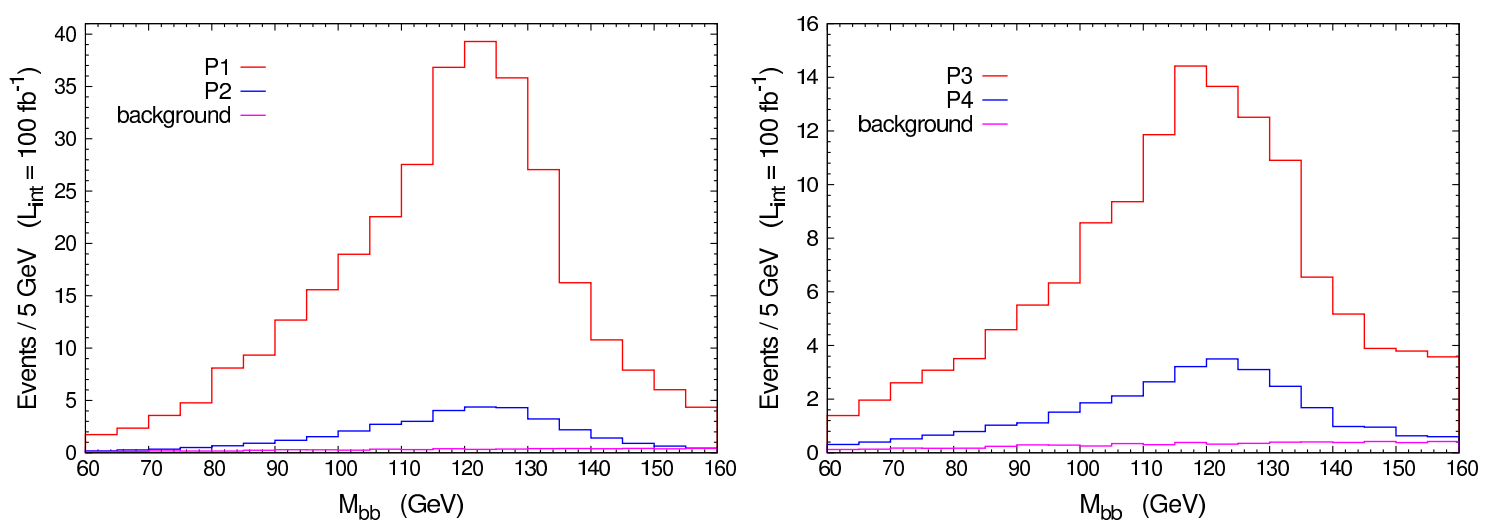

Figure 3. Spectra of $M_{b b}$ for the benchmark points P1 and P2 (left panel), and P3 and P4 (right panel).

\begin{tabular}{|c|c|c|c|c|c|c|c|c|}
\hline Point & P1 & P2 & P3 & P4 & P5 & P6 & P7 & P8 \\
\hline$\sigma_{\text {signal }}[\mathrm{fb}]$ & 3.1 & 0.35 & 1.3 & 0.30 & 0.45 & 2.0 & 0.56 & 0.46 \\
\hline accept. $\left[\times 10^{-3}\right]$ & 1.9 & 2.1 & 0.7 & 1.8 & 2.7 & 2.1 & 3.3 & 1.4 \\
\hline
\end{tabular}

Table 2. Cross sections in the signal region for the benchmark points $\mathrm{P} 1-\mathrm{P} 8$, as well as the corresponding acceptances.

$\tau$ 's, not by the cuts on jets (reducing the event rates by only $80 \%-90 \%$ ) nor by the cuts on $b$-jets. These acceptances are summarised in table 2 .

The $M_{b b}$ spectra are normalised to $100 \mathrm{fb}^{-1}$ integrated luminosity; since the event numbers per bin are small for P2 and P4, several $100 \mathrm{fb}^{-1}$ integrated luminosity will be required to see a statistically relevant number of signal events. Then, the signals of a $125 \mathrm{GeV}$ Higgs boson decaying into $b \bar{b}$ can be well visible above the background, even for $\mathrm{P} 2$ and P4 despite the large widths of the peak in this channel.

In figure 4 we display the spectra of $M_{b b}$ after the cuts described in section 3 for the points $\mathrm{P} 5-\mathrm{P} 8$. The cross sections in the signal region for these points are $\sim 0.45 \mathrm{fb}(\mathrm{P} 5)$, $\sim 2.0 \mathrm{fb}(\mathrm{P} 6), \sim 0.56 \mathrm{fb}(\mathrm{P} 7)$ and $\sim 0.46 \mathrm{fb}(\mathrm{P} 8)$, i.e. still above the background cross section in the signal region (see table 2). Again, event numbers in the signal regions are small for P5, P7 and P8; several $100 \mathrm{fb}^{-1}$ of integrated luminosity are required for a statistically relevant number of signal events. Given the acceptances of $\sim 1.4 \times 10^{-3}$ (for $\mathrm{P} 8)$ to $\sim 3.3 \times 10^{-3}$ (for $\mathrm{P} 7$ ), the signal rates — if visible — can provide at least rough information on the initial squark/gluon cross sections, and thus on the strongly interacting SUSY spectrum.

Additional observables, which can help to shed some light on the nature of the decay cascades, are the abundances of hard jets (with $P_{T}>100 \mathrm{GeV}$ ) and $b$-jets (with $P_{T}>$ $40 \mathrm{GeV}$ ) in the events which have passed the cuts. Both observables differ considerably for the various benchmark points. Extreme cases of high and low hard jet multiplicities are shown in the left panel of figure 5 for points P2 and P4; extreme cases of high and low $b$-jet multiplicities are shown in the right panel of figure 5 for points P3 and P8 (all normalised 

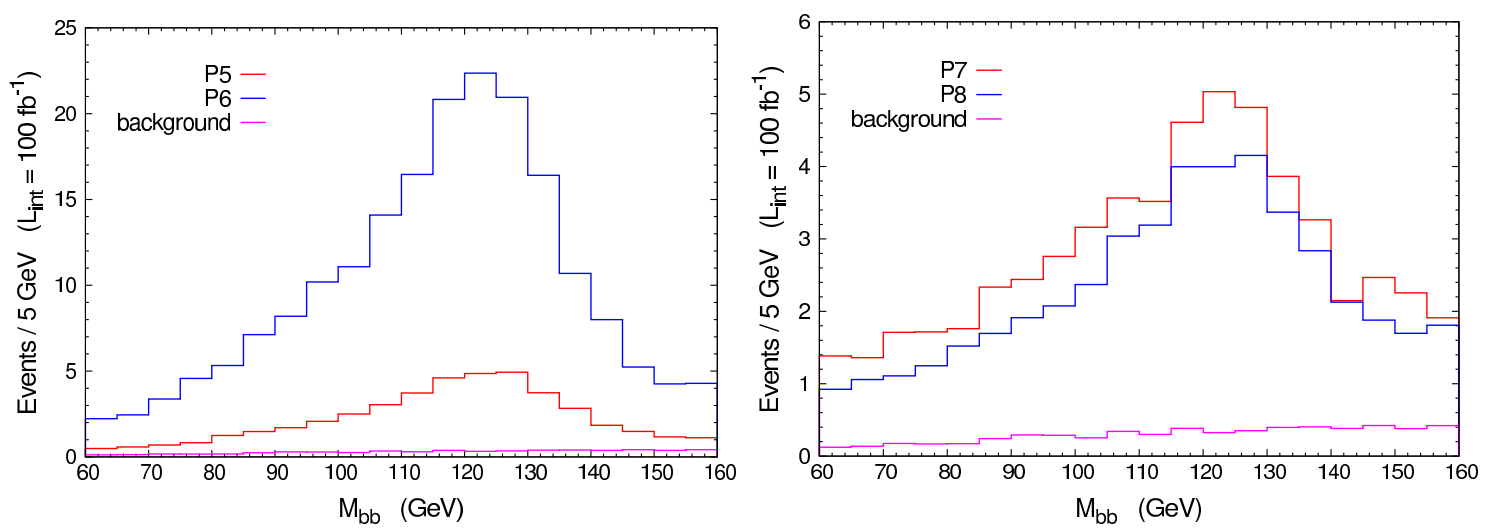

Figure 4. Spectra of $M_{b b}$ for the benchmark points P5 and P6 (left panel), and P7 and P8 (right panel).
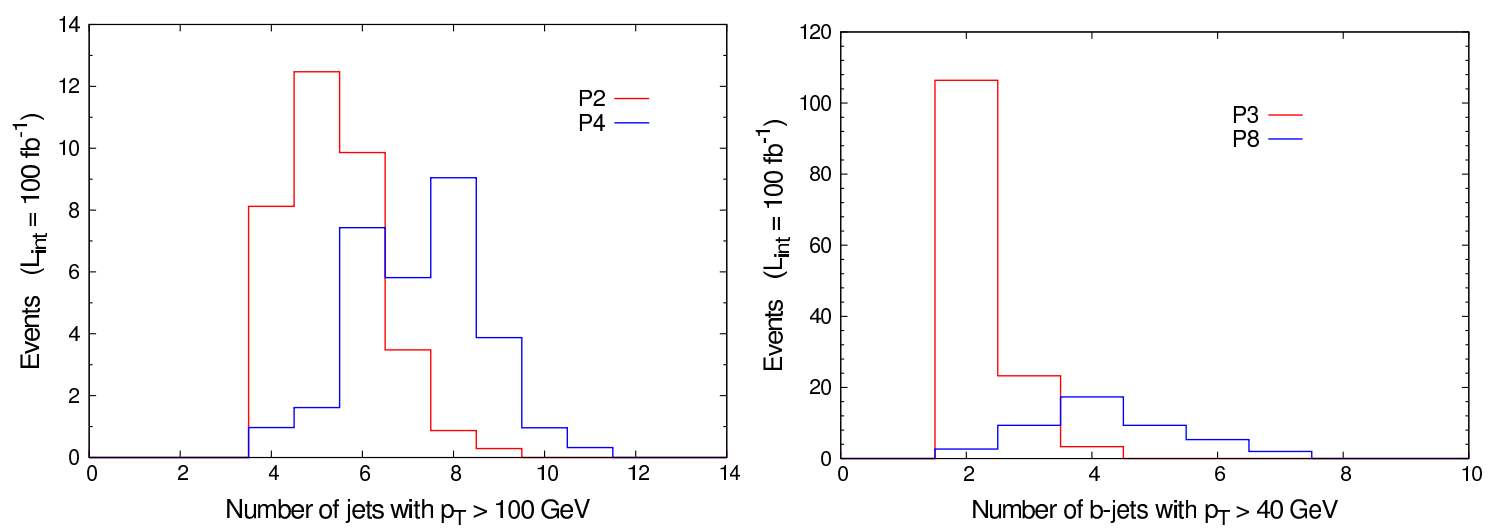

Figure 5. Hard jet (with $P_{T}>100 \mathrm{GeV}$ ) multiplicities for the benchmark points P2 and P4 (left panel), and $b$-jet multiplicities for the benchmark points P3 and P8 (right panel).

to $100 \mathrm{fb}^{-1}$ integrated luminosity).

The jet multiplicities provide information on the decay cascades: large hard jet multiplicities appear generally for benchmark points with gluinos lighter than squarks, such that squarks decay dominantly (or exclusively) via gluinos. Instead of showing diagrams of these multiplicities for all points, we find it convenient to define the ratio $R_{\text {hard }}$ of the number of events with 6 or more hard jets (with $P_{T}>100 \mathrm{GeV}$ ) to the number of events with 5 or less hard jets. This ratio is independent of the integrated luminosity and suffers somewhat less from statistical fluctuations than the absolute number of events per bin. (Of course, it still depends on the cuts applied, and even on the used jet algorithm.)

The $b$-jet multiplicities indicate whether top or bottom squarks appear in the gluino decay cascades, and are more pronounced if squarks decay via gluinos. Including two $b$-jets from one Higgs boson (as the other Higgs necessarily decays into two $\tau$ 's to satisfy our cuts), each event for P7 and P8 contains a priori six $b$-jets from the lowest order matrix element - not all of which are tagged, but QCD radiation can add more $b$ quark pairs. Again it is useful to define a ratio $R_{b \text {-jets }}$ of the number of events with 3 or more $b$-jets 


\begin{tabular}{|c|c|c|c|c|c|c|c|c|}
\hline & P1 & P2 & P3 & P4 & P5 & P6 & P7 & P8 \\
\hline$R_{\text {hard }}$ & 0.54 & 0.70 & 3.4 & 10.6 & 1.6 & 0.79 & 6.4 & 4.3 \\
\hline$R_{b-\text { jets }}$ & 0.09 & 0.09 & 0.25 & 0.37 & 1.0 & 0.63 & 6.8 & 16.3 \\
\hline
\end{tabular}

Table 3. The ratios $R_{\text {hard }}$ and $R_{b-\text { jets }}$ as defined in the text for the eight benchmark points P1-P8.

(with $P_{T}>40 \mathrm{GeV}$ ) to the number of events with exactly $2 b$-jets. The ratios $R_{\text {hard }}$ and $R_{b-\text { jets }}$ are summarised for the eight benchmark points in table 3 .

Despite the statistical fluctuations, the following trends can be observed:

- Points P1, P2, P5 and P6, with gluinos heavier than squarks (i.e. squarks decaying directly into a quark and the bino), have $R_{\text {hard }} \lesssim 2$ (actually $\lesssim 1$ except for P5 with gluinos decaying into top/stop); $R_{\text {hard }} \gtrsim 3$ indicates longer squark cascades via gluinos as for P3, P4, P7 and P8. Of course, $R_{\text {hard }}$ increases also with the squark masses, as is visible when comparing $\mathrm{P} 1 / \mathrm{P} 2$ and $\mathrm{P} 3 / \mathrm{P} 4$.

- Points P1, P2, P3 and P4 without stops/sbottoms in the gluino decay cascades all have $R_{b-\text { jets }} \lesssim 0.5$. Once gluinos can decay into stops/sbottoms, but for gluinos still heavier than squarks - as for P5 and P6 - we have $0.5 \lesssim R_{b-\text { jets }} \lesssim 1$. With stops/sbottoms in gluino decays and squarks heavier than gluinos (as for P7 and P8), we have $R_{b-\text { jets }} \gtrsim 6$.

Finally, we recall that points $\mathrm{P} 5 / \mathrm{P} 7$ with gluinos decaying via top/stop have sizeable $E_{T}^{\text {miss }}$ from leptonic top decays (see figure 2), which allows to distinguish them from points P6/P8, in which gluinos decay via bottom/sbottom pairs.

These (peculiar) features appear clear and easily distinguishable since the benchmark points correspond to simplified models with (mostly) 100\% branching fractions into given channels. Still, together with the signal rates, the discussed observables would give strong hints on the underlying sparticle spectrum.

\section{$5 \quad$ Summary and conclusions}

In the presence of a light singlino-like LSP in the NMSSM and an NLSP with a mass slightly above the threshold for the NLSP $\rightarrow$ LSP + Higgs decay, the $E_{T}^{\text {miss }}$ signature of sparticle production is considerably reduced. In these scenarios the upper bounds on squark/gluino masses from the run I at the LHC are alleviated, and search strategies not relying on large $E_{T}^{\text {miss }}$ would also be required for the run II at $13 \mathrm{TeV}$ c.m. energy.

We have proposed benchmark points corresponding to different squark/gluino masses and decay cascades, and studied the prospects for search strategies relying on two Higgs bosons in the final state. The proposed $2 b 2 \tau$ final state leads to large signal-to-background ratios for all masses and decay cascades considered here, but can still be optimised (considering, e.g., $2 b 2 \gamma$ or $4 b$ final states) after realistic detector simulations. This may be required for squark/gluino masses at or beyond the $1.4 / 1.5 \mathrm{TeV}$ range, where the small signal cross sections after our cuts would require several $100 \mathrm{fb}^{-1}$ of integrated luminosity; 
in this regime, in which the heavier supersymmetric cascades lead to more boosted Higgs, the signal significance could be further increased by using jet substructure techniques.

The scenario with a light singlino-like LSP in the NMSSM would also influence searches for direct stop/sbottom pair production, as well as searches for direct neutralino/chargino production. In all these cases, $E_{T}^{\text {miss }}$ would be reduced as well, and two Higgs bosons would appear instead in the decay cascades. Dedicated search strategies for these cases for the run II at the LHC remain to be devised.

\section{Acknowledgments}

U. E. and A. M. T. acknowledge support from European Union Initial Training Network INVISIBLES (PITN-GA-2011-289442). U. E. acknowledges support from the ERC advanced grant Higgs@LHC, and from the European Union Initial Training Network HiggsTools (PITN-GA-2012-316704).

Open Access. This article is distributed under the terms of the Creative Commons Attribution License (CC-BY 4.0), which permits any use, distribution and reproduction in any medium, provided the original author(s) and source are credited.

\section{References}

[1] ATLAS collaboration, Search for new phenomena in final states with large jet multiplicities and missing transverse momentum at $\sqrt{s}=8$ TeV proton-proton collisions using the ATLAS experiment, JHEP 10 (2013) 130 [arXiv:1308.1841] [INSPIRE].

[2] https://twiki.cern.ch/twiki/bin/view/AtlasPublic/SupersymmetryPublicResults.

[3] CMS collaboration, Search for new physics in the multijet and missing transverse momentum final state in proton-proton collisions at $\sqrt{s}=8 \mathrm{TeV}$, JHEP 06 (2014) 055 [arXiv: 1402.4770] [INSPIRE].

[4] J.L. Feng, Naturalness and the Status of Supersymmetry, Ann. Rev. Nucl. Part. Sci. 63 (2013) 351 [arXiv:1302.6587] [InSPIRE].

[5] N. Craig, The State of Supersymmetry after Run I of the LHC, arXiv:1309.0528 [INSPIRE].

[6] I. Melzer-Pellmann and P. Pralavorio, Lessons for SUSY from the LHC after the first run, Eur. Phys. J. C 74 (2014) 2801 [arXiv:1404.7191] [InSPIRE].

[7] E. Halkiadakis, G. Redlinger and D. Shih, Status and Implications of Beyond-the-Standard-Model Searches at the LHC, Ann. Rev. Nucl. Part. Sci. 64 (2014) 319 [arXiv: 1411.1427] [INSPIRE].

[8] ATLAS collaboration, Search for squarks and gluinos with the ATLAS detector in final states with jets and missing transverse momentum using $\sqrt{s}=8$ TeV proton-proton collision data, JHEP 09 (2014) 176 [arXiv:1405.7875] [INSPIRE].

[9] J. Fan, M. Reece and J.T. Ruderman, Stealth Supersymmetry, JHEP 11 (2011) 012 [arXiv:1105.5135] [INSPIRE].

[10] M. Lisanti, P. Schuster, M. Strassler and N. Toro, Study of LHC Searches for a Lepton and Many Jets, JHEP 11 (2012) 081 [arXiv:1107.5055] [INSPIRE]. 
[11] J. Fan, M. Reece and J.T. Ruderman, A Stealth Supersymmetry Sampler, JHEP 07 (2012) 196 [arXiv:1201.4875] [INSPIRE].

[12] M. Baryakhtar, N. Craig and K. Van Tilburg, Supersymmetry in the Shadow of Photini, JHEP 07 (2012) 164 [arXiv:1206.0751] [INSPIRE].

[13] J.A. Evans, Y. Kats, D. Shih and M.J. Strassler, Toward Full LHC Coverage of Natural Supersymmetry, JHEP 07 (2014) 101 [arXiv:1310.5758] [INSPIRE].

[14] U. Ellwanger and A.M. Teixeira, NMSSM with a singlino LSP: possible challenges for searches for supersymmetry at the LHC, JHEP 10 (2014) 113 [arXiv:1406.7221] [INSPIRE].

[15] U. Ellwanger, C. Hugonie and A.M. Teixeira, The Next-to-Minimal Supersymmetric Standard Model, Phys. Rept. 496 (2010) 1 [arXiv:0910.1785] [INSPIRE].

[16] J.E. Kim and H.P. Nilles, The mu Problem and the Strong CP Problem, Phys. Lett. B 138 (1984) 150 [INSPIRE].

[17] L.J. Hall, D. Pinner and J.T. Ruderman, A Natural SUSY Higgs Near 126 GeV, JHEP 04 (2012) 131 [arXiv: 1112.2703] [INSPIRE].

[18] U. Ellwanger, A Higgs boson near $125 \mathrm{GeV}$ with enhanced di-photon signal in the NMSSM, JHEP 03 (2012) 044 [arXiv: 1112.3548] [INSPIRE].

[19] A. Arvanitaki and G. Villadoro, A Non Standard Model Higgs at the LHC as a Sign of Naturalness, JHEP 02 (2012) 144 [arXiv:1112.4835] [INSPIRE].

[20] S.F. King, M. Muhlleitner and R. Nevzorov, NMSSM Higgs Benchmarks Near $125 \mathrm{GeV}$, Nucl. Phys. B 860 (2012) 207 [arXiv:1201.2671] [INSPIRE].

[21] Z. Kang, J. Li and T. Li, On Naturalness of the MSSM and NMSSM, JHEP 11 (2012) 024 [arXiv:1201.5305] [INSPIRE].

[22] J.-J. Cao, Z.-X. Heng, J.M. Yang, Y.-M. Zhang and J.-Y. Zhu, A SM-like Higgs near 125 GeV in low energy SUSY: a comparative study for MSSM and NMSSM, JHEP 03 (2012) 086 [arXiv:1202.5821] [INSPIRE].

[23] ATLAS collaboration, Search for massive particles in multijet signatures with the ATLAS detector in $\sqrt{s}=8 \mathrm{TeV}$ pp collisions at the LHC, ATLAS-CONF-2013-091 (2013).

[24] CMS Collaboration, Search for light- and heavy-flavor three-jet resonances in multijet final states at $8 \mathrm{TeV}$, CMS-PAS-EXO-12-049 (Search for light- and heavy-flavor three-jet resonances in multijet final states at $8 \mathrm{TeV}$ ).

[25] CMS collaboration, Search for pair-produced dijet resonances in four-jet final states in pp collisions at $\sqrt{s}=7 \mathrm{TeV}$, Phys. Rev. Lett. 110 (2013) 141802 [arXiv:1302.0531] [INSPIRE].

[26] CMS collaboration, Search for microscopic black holes in pp collisions at $\sqrt{s}=8 \mathrm{TeV}$, JHEP 07 (2013) 178 [arXiv: 1303.5338] [INSPIRE].

[27] S. Dimopoulos, M. Dine, S. Raby and S.D. Thomas, Experimental signatures of low-energy gauge mediated supersymmetry breaking, Phys. Rev. Lett. 76 (1996) 3494 [hep-ph/9601367] [INSPIRE].

[28] S. Ambrosanio, G.L. Kane, G.D. Kribs, S.P. Martin and S. Mrenna, Search for supersymmetry with a light gravitino at the Fermilab Tevatron and CERN LEP colliders, Phys. Rev. D 54 (1996) 5395 [hep-ph/9605398] [INSPIRE].

[29] I. Hinchliffe, F.E. Paige, M.D. Shapiro, J. Soderqvist and W. Yao, Precision SUSY measurements at CERN LHC, Phys. Rev. D 55 (1997) 5520 [hep-ph/9610544] [INSPIRE]. 
[30] K.T. Matchev and S.D. Thomas, Higgs and $Z$ boson signatures of supersymmetry, Phys. Rev. D 62 (2000) 077702 [hep-ph/9908482] [INSPIRE].

[31] A. Datta, A. Djouadi, M. Guchait and F. Moortgat, Detection of MSSM Higgs bosons from supersymmetric particle cascade decays at the LHC, Nucl. Phys. B 681 (2004) 31 [hep-ph/0303095] [INSPIRE].

[32] P. Bandyopadhyay, A. Datta and B. Mukhopadhyaya, Signatures of gaugino mass non-universality in cascade Higgs production at the LHC, Phys. Lett. B 670 (2008) 5 [arXiv:0806.2367] [INSPIRE].

[33] K. Huitu et al., Search for Higgs Bosons in SUSY Cascades in CMS and Dark Matter with Non-universal Gaugino Masses, Eur. Phys. J. C 58 (2008) 591 [arXiv:0808.3094] [INSPIRE].

[34] P. Bandyopadhyay, Probing non-universal gaugino masses via Higgs boson production under SUSY cascades at the LHC: A Detailed study, JHEP 07 (2009) 102 [arXiv:0811.2537] [INSPIRE].

[35] A.C. Fowler and G. Weiglein, Precise Predictions for Higgs Production in Neutralino Decays in the Complex MSSM, JHEP 01 (2010) 108 [arXiv:0909.5165] [INSPIRE].

[36] P. Meade, M. Reece and D. Shih, Prompt Decays of General Neutralino NLSPs at the Tevatron, JHEP 05 (2010) 105 [arXiv:0911.4130] [INSPIRE].

[37] M. Asano, H.D. Kim, R. Kitano and Y. Shimizu, Natural Supersymmetry at the LHC, JHEP 12 (2010) 019 [arXiv: 1010.0692] [INSPIRE].

[38] J. Thaler and Z. Thomas, Goldstini Can Give the Higgs a Boost, JHEP 07 (2011) 060 [arXiv:1103.1631] [INSPIRE].

[39] S. Gori, P. Schwaller and C.E.M. Wagner, Search for Higgs Bosons in SUSY Cascade Decays and Neutralino Dark Matter, Phys. Rev. D 83 (2011) 115022 [arXiv:1103.4138] [INSPIRE].

[40] J.T. Ruderman and D. Shih, General Neutralino NLSPs at the Early LHC, JHEP 08 (2012) 159 [arXiv: 1103.6083] [INSPIRE].

[41] Y. Kats, P. Meade, M. Reece and D. Shih, The Status of GMSB After 1/fb at the LHC, JHEP 02 (2012) 115 [arXiv:1110.6444] [INSPIRE].

[42] H. Baer, V. Barger, A. Lessa, W. Sreethawong and X. Tata, Wh plus missing- $E_{T}$ signature from gaugino pair production at the LHC, Phys. Rev. D 85 (2012) 055022 [arXiv: 1201.2949] [INSPIRE].

[43] D. Ghosh, M. Guchait and D. Sengupta, Higgs Signal in Chargino-Neutralino Production at the LHC, Eur. Phys. J. C 72 (2012) 2141 [arXiv:1202.4937] [INSPIRE].

[44] A. Belyaev, J.P. Hall, S.F. King and P. Svantesson, Novel gluino cascade decays in $E_{6}$ inspired models, Phys. Rev. D 86 (2012) 031702 [arXiv:1203.2495] [INSPIRE].

[45] P. Byakti and D. Ghosh, Magic Messengers in Gauge Mediation and signal for $125 \mathrm{GeV}$ boosted Higgs boson, Phys. Rev. D 86 (2012) 095027 [arXiv: 1204.0415] [INSPIRE].

[46] K. Howe and P. Saraswat, Excess Higgs Production in Neutralino Decays, JHEP 10 (2012) 065 [arXiv:1208.1542] [InSPIRE].

[47] A. Belyaev, J.P. Hall, S.F. King and P. Svantesson, Discovering $E_{6}$ supersymmetric models in gluino cascade decays at the LHC, Phys. Rev. D 87 (2013) 035019 [arXiv:1211.1962] [INSPIRE]. 
[48] A. Arbey, M. Battaglia and F. Mahmoudi, Higgs Production in Neutralino Decays in the MSSM - The LHC and a Future $e^{+} e^{-}$Collider, Eur. Phys. J. C 75 (2015) 108 [arXiv: 1212.6865] [INSPIRE].

[49] A. Bharucha, S. Heinemeyer and F. von der Pahlen, Direct Chargino-Neutralino Production at the LHC: Interpreting the Exclusion Limits in the Complex MSSM,

Eur. Phys. J. C 73 (2013) 2629 [arXiv:1307.4237] [InSPIRE].

[50] T. Han, S. Padhi and S. Su, Electroweakinos in the Light of the Higgs Boson, Phys. Rev. D 88 (2013) 115010 [arXiv:1309.5966] [inSPIRE].

[51] F. Yu, Anatomizing Exotic Production of the Higgs Boson, Phys. Rev. D 90 (2014) 015009 [arXiv: 1404.2924] [INSPIRE].

[52] F. Franke and H. Fraas, Production and decay of neutralinos in the next-to-minimal supersymmetric standard model, Z. Phys. C 72 (1996) 309 [hep-ph/9511275] [INSPIRE].

[53] U. Ellwanger and C. Hugonie, Neutralino cascades in the $(M+1) S S M$, Eur. Phys. J. C 5 (1998) 723 [hep-ph/9712300] [INSPIRE].

[54] S.Y. Choi, D.J. Miller and P.M. Zerwas, The Neutralino sector of the next-to-minimal supersymmetric standard model, Nucl. Phys. B 711 (2005) 83 [hep-ph/0407209] [InSPIRE].

[55] K. Cheung and T.-J. Hou, Light Pseudoscalar Higgs boson in Neutralino Decays in the Next-to-Minimal Supersymmetric Standard Model, Phys. Lett. B 674 (2009) 54 [arXiv:0809.1122] [INSPIRE].

[56] O. Stal and G. Weiglein, Light NMSSM Higgs bosons in SUSY cascade decays at the LHC, JHEP 01 (2012) 071 [arXiv:1108.0595] [INSPIRE].

[57] D. Das, U. Ellwanger and A.M. Teixeira, Modified Signals for Supersymmetry in the NMSSM with a Singlino-like LSP, JHEP 04 (2012) 067 [arXiv:1202.5244] [INSPIRE].

[58] D.G. Cerdeño, P. Ghosh, C.B. Park and M. Peiró, Collider signatures of a light NMSSM pseudoscalar in neutralino decays in the light of LHC results, JHEP 02 (2014) 048 [arXiv: 1307.7601] [INSPIRE].

[59] G.D. Kribs, A. Martin, T.S. Roy and M. Spannowsky, Discovering the Higgs Boson in New Physics Events using Jet Substructure, Phys. Rev. D 81 (2010) 111501 [arXiv:0912.4731] [INSPIRE].

[60] G.D. Kribs, A. Martin, T.S. Roy and M. Spannowsky, Discovering Higgs Bosons of the MSSM using Jet Substructure, Phys. Rev. D 82 (2010) 095012 [arXiv:1006.1656] [INSPIRE].

[61] B. Bhattacherjee, A. Chakraborty, D. Kumar Ghosh and S. Raychaudhuri, Using Jet Substructure at the LHC to Search for the Light Higgs Bosons of the CP-Violating MSSM, Phys. Rev. D 86 (2012) 075012 [arXiv: 1204.3369] [INSPIRE].

[62] U. Ellwanger, J.F. Gunion and C. Hugonie, NMHDECAY: A Fortran code for the Higgs masses, couplings and decay widths in the NMSSM, JHEP 02 (2005) 066 [hep-ph/0406215] [INSPIRE].

[63] U. Ellwanger and C. Hugonie, NMHDECAY 2.0: An Updated program for sparticle masses, Higgs masses, couplings and decay widths in the NMSSM, Comput. Phys. Commun. 175 (2006) 290 [hep-ph/0508022] [INSPIRE].

[64] M. Drees, H. Dreiner, D. Schmeier, J. Tattersall and J.S. Kim, CheckMATE: Confronting 
your Favourite New Physics Model with LHC Data,

Comput. Phys. Commun. 187 (2014) 227 [arXiv:1312.2591] [INSPIRE].

[65] W. Beenakker, R. Hopker, M. Spira and P.M. Zerwas, Squark and gluino production at hadron colliders, Nucl. Phys. B 492 (1997) 51 [hep-ph/9610490] [INSPIRE].

[66] W. Beenakker, R. Hopker and M. Spira, PROSPINO: A Program for the production of supersymmetric particles in next-to-leading order QCD, hep-ph/9611232 [INSPIRE].

[67] J. Alwall, M. Herquet, F. Maltoni, O. Mattelaer and T. Stelzer, MadGraph 5 : Going Beyond, JHEP 06 (2011) 128 [arXiv:1106.0522] [INSPIRE].

[68] T. Sjöstrand, S. Mrenna and P.Z. Skands, PYTHIA 6.4 Physics and Manual, JHEP 05 (2006) 026 [hep-ph/0603175] [INSPIRE].

[69] DELPHES 3 collaboration, J. de Favereau et al., DELPHES 3, A modular framework for fast simulation of a generic collider experiment, JHEP 02 (2014) 057 [arXiv:1307.6346] [INSPIRE].

[70] M. Cacciari, G.P. Salam and G. Soyez, FastJet User Manual, Eur. Phys. J. C 72 (2012) 1896 [arXiv:1111.6097] [InSPIRE].

[71] M. Cacciari, G.P. Salam and G. Soyez, The Anti-k(t) jet clustering algorithm, JHEP 04 (2008) 063 [arXiv: 0802.1189] [inSPIRE].

[72] E. Conte, B. Fuks and G. Serret, MadAnalysis 5, A User-Friendly Framework for Collider Phenomenology, Comput. Phys. Commun. 184 (2013) 222 [arXiv:1206.1599] [INSPIRE].

[73] E. Conte and B. Fuks, MadAnalysis 5: status and new developments, J. Phys. Conf. Ser. 523 (2014) 012032 [arXiv: 1309.7831] [INSPIRE]. 